\title{
Correlation Properties of (Discrete) Fractional Gaussian Noise and Fractional Brownian Motion
}

\author{
Didier Delignières \\ EA 2991 Movement to Health, Euromov, University of Montpellier, 34090 Montpellier, France \\ Correspondence should be addressed to Didier Delignières; didier.delignieres@univ-montp1.fr
}

Received 13 April 2015; Revised 9 July 2015; Accepted 2 August 2015

Academic Editor: Paul Bogdan

Copyright (C) 2015 Didier Delignières. This is an open access article distributed under the Creative Commons Attribution License, which permits unrestricted use, distribution, and reproduction in any medium, provided the original work is properly cited.

The fractional Gaussian noise/fractional Brownian motion framework ( $\mathrm{fGn} / \mathrm{fBm}$ ) has been widely used for modeling and interpreting physiological and behavioral data. The concept of $1 / f$ noise, reflecting a kind of optimal complexity in the underlying systems, is of central interest in this approach. It is generally considered that $\mathrm{fGn}$ and $\mathrm{fBm}$ represent a continuum, punctuated by the boundary of "ideal" $1 / f$ noise. In the present paper, we focus on the correlation properties of discrete-time versions of these processes (dfGn and $\mathrm{dfBm}$ ). We especially derive a new analytical expression of the autocorrelation function (ACF) of dfBm. We analyze the limit behavior of $\mathrm{dfGn}$ and $\mathrm{dfBm}$ when they approach their upper and lower limits, respectively. We show that, as $H$ approaches 1, the ACF of dfGn tends towards 1 at all lags, suggesting that dfGn series tend towards straight line. Conversely, as $H$ approaches 0 , the $\mathrm{ACF}$ of $\mathrm{dfBm}$ tends towards 0 at all lags, suggesting that $\mathrm{dfBm}$ series tend towards white noise. These results reveal a severe breakdown of correlation properties around the $1 / f$ boundary and challenge the idea of a smooth transition between dfGn and $\mathrm{dfBm}$ processes. We discuss the implications of these findings for the application of the $\mathrm{dfGn} / \mathrm{dfBm}$ model to experimental series, in terms of theoretical interpretation and modeling.

\section{Introduction}

During the last decades, there has been a considerable interest in the use of stochastic fractal models for interpreting physiological or behavioral data. These models have been applied to various processes, including in the physiological domain heart-beat variability $[1,2]$, brain activity $[3,4]$, respiratory fluctuations [5], or blood flow [6]. Studies on sensorimotor processes included isometric force production [7], visual search [8], finger tapping $[9,10]$, or bimanual coordination [11]. Recent research has motivated the use of fractal models in medical and rehabilitation devices, in order to conceive efficient noninvasive stimuli for living organisms [12].

The most popular formalization of this approach refers to the concepts of fractional Brownian motion $(\mathrm{fBm})$ and fractional Gaussian noise ( $\mathrm{fGn}$ ), initially introduced by Mandelbrot and Van Ness [13]. fBm and fGn represent two families of correlated stochastic processes, possessing powerful statistical properties, which seem able to provide relevant models for a wide range of empirical observations in various domains.
The success of the $\mathrm{fGn} / \mathrm{fBm}$ concept is mainly related to the presence, in some parts the model, of series possessing long-range correlation properties. Long-range correlations are characterized by a very slow decay of the autocorrelation function and suggest that the system possesses a long-term, multiscale memory of its previous states that affects its current behavior. Long-range correlations have been popularized through the concept of $1 / f$ noise, thought of as a narrow range in the $\mathrm{fGn} / \mathrm{fBm}$ model where long-range correlations reach maximal values. $1 / f$-like fluctuations have been discovered in the behavior of a number of physiological and behavioral systems (see references above) and also in several physical and nonliving systems [14-16]. In living systems, $1 / f$ fluctuations have been evidenced in the behavior of young, healthy, and perennial organisms, and aging or disease seems to be characterized by an alteration of this long-range correlated behavior $[1,17]$. This ubiquity has represented a very intriguing phenomenon in various scientific domains and its understanding has been a major challenge during the last decades. Long-range correlations are supposed to reflect the complexity of the underlying system, and $1 / f$ noise 
a kind of optimal complexity, a compromise between order and disorder $[1,2]$.

Most analysis methods of long-range correlated processes are based on the statistical properties of $\mathrm{fGn}$ and $\mathrm{fBm}$. However, we think that a number of issues about these processes remain unclear, often leading to erroneous interpretations. In this paper, we propose a formal analysis of the correlational properties of $\mathrm{fBm}$ and $\mathrm{fGn}$, focusing on the limit behavior of these processes when approaching their definition boundaries. Considering the state of the art about this model, our main aim is to derive an analytical expression of the autocorrelation function of the discrete version of $\mathrm{fBm}$.

\section{The $\mathbf{f B m} / \mathbf{f G n}$ Model}

Fractional Brownian motion ( $\mathrm{fBm}$ ), denoted by $B_{t}^{H}$, was initially introduced by Mandelbrot and Van Ness [13] as a continuous stochastic process $t$ ranging over all nonnegative real values. A fundamental property of $\mathrm{fBm}$ is that in such process variance is a power function of the time span over which it is computed:

$$
\operatorname{Var}\left(B_{t}^{H}\right) \propto t^{2 H}
$$

where $H$ is the Hurst exponent, which can take any real value within the interval $] 0,1[$.

In experimental and engineering applications, researchers often deal with sampled data, and such sampling leads to a discrete-time version of $\mathrm{fBm}, B_{i}^{H}, i \in \mathbb{N}$, referred to as $\mathrm{dfBm}$. We focus in this paper on this discrete version, which corresponds to most analyses performed in physiological and behavioral experiments. Discrete-time fractional Gaussian noise (dfGn), denoted by $G_{i}^{H}$, is defined as the series of increments in a $\operatorname{dfBm}\left(G_{i}^{H}=B_{i}^{H}-B_{i-1}^{H}\right)$.

By definition, a dfGn is the difference of a dfBm, and conversely the cumulative sum of $\mathrm{dfG}$ gives a $\mathrm{dfBm}$. Each $\mathrm{dfBm}$ series is then related to a specific $\mathrm{dfG}$, and both are characterized by the same $H$ exponent. dfBm are nonstationary processes, as suggested by (1), whereas dfGn series present stationary mean and variance over time.

For $H=0.5, B_{i}^{H}$ corresponds to ordinary Brownian motion, its variance is proportional to series length (normal diffusion), and $G_{i}^{H}$ is a white noise process. For $H<0.5$, $B_{i}^{H}$ is subdiffusive, and successive values in $G_{i}^{H}$ are negatively correlated (antipersistent). In contrast, for $H>0.5, B_{i}^{H}$ is overdiffusive and successive values in $G_{i}^{H}$ are positively correlated (persistent).

$\mathrm{dfBm}$ and $\mathrm{dfGn}$ are characterized by some essential basic properties [18]. Consider a dfBm process $B_{i}^{H}, i=$ $0,1,2, \ldots, N$, and its corresponding $\operatorname{dfGn} G_{i}^{H}, i=1,2, \ldots, N$. $B_{i}^{H}$ is the sum of $i$ first $G_{i}^{H}\left(B_{i}^{H}=G_{1}^{H}+G_{2}^{H}+\cdots+G_{i}^{H}\right)$. Let $\sigma^{2}$ be the variance of the dfGn process $\left(\sigma^{2}=\operatorname{Var}\left(G_{i}^{H}\right)=\right.$ $\left.E\left[\left(B_{i}^{H}-B_{i-1}^{H}\right)^{2}\right]\right)$. For large $N$, the expected value of dfBm is zero $\left(E\left(B_{i}^{H}\right)=0\right)$, as well as the expected values of differences within $\mathrm{dfBm}\left(E\left(B_{i}^{H}-B_{j}^{H}\right)=0\right)$. dfBm is characterized by self-similarity properties, which can be expressed at different levels. First, and similar to the property expressed in (1) for
$\mathrm{fBm}$, the variance of a sample path of length $n$ of a $\mathrm{dfBm}$ is a power function of $n$ :

$$
\operatorname{Var}\left(B_{n}^{H}\right)=\operatorname{Var}\left\{B_{0}^{H}, B_{1}^{H}, \ldots, B_{n}^{H}\right\}=C n^{2 H},
$$

where $C$ is the scaling coefficient, depending on both $H$ and $\sigma^{2}$.

Another self-similarity property characterizes the variance of a fixed lag difference between $\mathrm{dfBm}$ values:

$$
\operatorname{Var}\left(B_{i}^{H}-B_{j}^{H}\right)=E\left[\left(B_{i}^{H}-B_{j}^{H}\right)^{2}\right]=\sigma^{2}(i-j)^{2 H} .
$$

The autocovariance function of a dfGn is given by $[18,19]$

$$
E\left(G_{0}^{H} G_{k}^{H}\right)=\frac{\sigma^{2}}{2}\left(|k+1|^{2 H}+|k-1|^{2 H}-2|k|^{2 H}\right),
$$

yielding a simple expression for the autocorrelation function of dfGn:

$$
\rho_{\mathrm{dfGn}}(k)=\frac{1}{2}\left(|k+1|^{2 H}+|k-1|^{2 H}-2|k|^{2 H}\right) .
$$

\section{The Autocorrelation Function of dfBm}

The main aim of this paper is to derive an expression of the autocorrelation function of $\mathrm{fBm}$ in the discrete-time case. A well-known expression of the expected covariance of a continuous-time $\mathrm{fBm}$ series between two times $t$ and $s$ was given by Beran [18]:

$$
E\left[B_{t}^{H} B_{s}^{H}\right]=\frac{\sigma^{2}}{2}\left[t^{2 H}+s^{2 H}-|s-t|^{2 H}\right] .
$$

This autocovariance function depends explicitly on $t$ and $s$, and not only on $(t-s)$, fBm being nonstationary [20]. Here we aim at deriving an approximate expression of the autocorrelation in the discrete-time case, depending only on $k$ and series length.

The autocorrelation of lag $k$ of $\mathrm{dfBm}$ is given by the ratio between the corresponding covariance and the variance of the process:

$$
\begin{aligned}
\rho\left(B_{i}^{H}, B_{i+k}^{H}\right)=\frac{\gamma\left(B_{i}^{H}, B_{i+k}^{H}\right)}{\operatorname{Var}\left(B_{i}^{H}\right)}=\frac{E\left[\left(B_{i}^{H} B_{i+k}^{H}\right)^{2}\right]}{\operatorname{Var}\left(B_{i}^{H}\right)} \\
=\frac{E\left[\left(B_{i}^{H}\right)^{2}\right]+E\left[\left(B_{i+k}^{H}\right)^{2}\right]-E\left[\left(B_{i+k}^{H}-B_{i}^{H}\right)^{2}\right]}{2 \operatorname{Var}\left(B_{i}^{H}\right)} \\
=\frac{E\left[\left(B_{i}^{H}\right)^{2}\right]+E\left[\left(B_{i+k}^{H}\right)^{2}\right]-\sigma^{2} k^{2 H}}{2 \operatorname{Var}\left(B_{i}^{H}\right)},
\end{aligned}
$$

which could be approximated for large $i$ as

$$
\rho\left(B_{i}^{H}, B_{i+k}^{H}\right)=1-\frac{\sigma^{2} k^{2 H}}{2 \operatorname{Var}\left(B_{i}^{H}\right)} .
$$

For simplifying (8), we need an exact expression of $\operatorname{Var}\left(B_{i}^{H}\right)$, as a function of $\sigma^{2}$ and $H$. Consider a sample path of 
$B_{i}^{H}$ of length $n$. The variance of this sample path, considering the nonbiased estimator, can be expressed as

$$
\begin{aligned}
\operatorname{Var}\left(B_{i}^{H}\right) & =\frac{n}{n-1} E\left[B_{i}^{H}-E\left(B_{i}^{H}\right)\right]^{2} \\
& =\frac{n}{n-1} E\left(B_{i}^{H}-\frac{1}{n} \sum_{i=0}^{n-1} B_{i}^{H}\right)^{2} .
\end{aligned}
$$

In the simplest case $(n=2)$, we have

$$
\begin{aligned}
& \operatorname{Var}\left(B_{i}^{H}\right)=2 E\left[B_{i}^{H}-E\left(B_{i}^{H}\right)\right]^{2}=\frac{2}{2} \\
& \cdot E\left\{\left[\left(B_{i}^{H}-\frac{B_{i}^{H}+B_{i+1}^{H}}{2}\right)^{2}\right]\right. \\
& \left.+E\left[\left(B_{i+1}^{H}-\frac{B_{i}^{H}+B_{i+1}^{H}}{2}\right)^{2}\right]\right\}=E\left[\left(B_{i}^{H}\right)^{2}\right] \\
& +E\left[\left(B_{i+1}^{H}\right)^{2}\right]+2 E\left[\left(\frac{B_{i}^{H}+B_{i+1}^{H}}{2}\right)^{2}\right] \\
& -2 E\left[B_{i}^{H}\left(\frac{B_{i}^{H}+B_{i+1}^{H}}{2}\right)\right] \\
& -2 E\left[B_{i+1}^{H}\left(\frac{B_{i}^{H}+B_{i+1}^{H}}{2}\right)\right] .
\end{aligned}
$$

Developing the preceding equation, we get

$$
\begin{aligned}
\operatorname{Var}\left(B_{2}^{H}\right)= & \frac{1}{2} E\left[\left(B_{i}^{H}\right)\right]^{2}+\frac{1}{2} E\left[\left(B_{i+1}^{H}\right)^{2}\right] \\
& -E\left(B_{i}^{H} B_{i+1}^{H}\right) .
\end{aligned}
$$

And replacing $B_{i+1}^{H}$ by $B_{i}^{H}+G_{i}^{H}$,

$$
\begin{aligned}
\operatorname{Var}\left(B_{i}^{H}\right)= & \frac{1}{2} E\left[\left(B_{i}^{H}\right)\right]^{2}+\frac{1}{2} E\left[\left(B_{i}^{H}+G_{i}^{H}\right)^{2}\right] \\
& -E\left[B_{i}^{H}\left(B_{i}^{H}+G_{i}^{H}\right)\right] \\
= & \frac{1}{2} E\left[\left(B_{i}^{H}\right)^{2}\right]+\frac{1}{2} E\left[\left(B_{i}^{H}\right)^{2}\right] \\
& +\frac{1}{2} E\left[\left(G_{i}^{H}\right)^{2}\right]+E\left(B_{i}^{H} G_{i}^{H}\right) \\
& -E\left[\left(B_{i}^{H}\right)^{2}\right]-E\left(B_{i}^{H} G_{i}^{H}\right) .
\end{aligned}
$$

All terms containing $B_{i}^{H}$ vanishing in the previous equation, we obtain

$$
\operatorname{Var}\left(B_{i}^{H}\right)=\frac{1}{2} E\left[\left(G_{i}^{H}\right)^{2}\right]
$$

Using similar calculations for $n=3, n=4$, and $n=5$, we get, respectively,

$$
\begin{array}{ll}
n=3 & \operatorname{Var}\left(B_{i}^{H}\right)=\frac{1}{6}\left\{4 E\left[\left(G_{i}^{H}\right)^{2}\right]+2 E\left(G_{i}^{H} G_{i+1}^{H}\right)\right\}, \\
n=4 & \operatorname{Var}\left(B_{i}^{H}\right)=\frac{1}{12}\left\{10 E\left[\left(G_{i}^{H}\right)^{2}\right]+8 E\left(G_{i}^{H} G_{i+1}^{H}\right)+2 E\left(G_{i}^{H} G_{i+2}^{H}\right)\right\}, \\
n=5 & \operatorname{Var}\left(B_{i}^{H}\right)=\frac{1}{20}\left\{20 E\left[\left(G_{i}^{H}\right)^{2}\right]+20 E\left(G_{i}^{H} G_{i+1}^{H}\right)+8 E\left(G_{i}^{H} G_{i+2}^{H}\right)+2 E\left(G_{i}^{H} G_{i+3}^{H}\right)\right\} .
\end{array}
$$

Replacing $E\left[\left(G_{i}^{H}\right)^{2}\right]$ by $\sigma^{2}$ and incorporating (4), we finally obtain

$$
\begin{array}{ll}
n=2 & \operatorname{Var}\left(B_{i}^{H}\right)=\frac{1}{2} \sigma^{2}, \\
n=3 & \operatorname{Var}\left(B_{i}^{H}\right)=\frac{1}{6} \sigma^{2}\left[2+2^{2 H}\right], \\
n=4 & \operatorname{Var}\left(B_{i}^{H}\right)=\frac{1}{12} \sigma^{2}\left[3+2\left(2^{2 H}\right)+3^{2 H}\right],
\end{array}
$$$$
n=5
$$$$
\operatorname{Var}\left(B_{i}^{H}\right)=\frac{1}{20} \sigma^{2}\left[4+3\left(2^{2 H}\right)+2\left(3^{2 H}\right)+4^{2 H}\right],
$$

One can easily show that if (18) is true for $n$, then it also works for $n+1$. In passing, we obtain an exact expression for the diffusion coefficient $C(2)$ :

$$
C=\frac{\operatorname{Var}\left(B_{n}^{H}\right)}{n^{2 H}}=\frac{\sigma^{2}}{(n-1) n^{2 H+1}} \sum_{i=1}^{n-1}\left[(n-i) i^{2 H}\right]
$$


Combining (8) and (18), we get an expression for the autocorrelation function of $\mathrm{dfBm}$ :

$$
\begin{aligned}
\rho_{\mathrm{dfBm}}(k) & =1-\frac{\sigma^{2} k^{2 H}}{2 \operatorname{Var}\left(B_{n}^{H}\right)} \\
& =1-\frac{n(n-1) k^{2 H}}{2 \sum_{i=1}^{n-1}\left[(n-i) i^{2 H}\right]} .
\end{aligned}
$$

\section{Limit Behaviors of $\mathrm{dfBm}$ and dfGn}

Equations (5) and (20) allow analyzing the limit behaviors of dfBm and dfGn, when $H$ reaches the limits of the interval ]0,1[. Considering the upper limit of the $H$ interval, (5) predicts that when $H$ tends towards $1, \rho_{\mathrm{dfGn}}(k)$ tends towards 1.0 for all $k \geq 0$. In other words, when $H$ tends towards $1, G_{i}^{H}$ tends towards a straight line.

One can easily show that

$$
\sum_{k=1}^{n-1}(n-k)=\frac{n(n-1)}{2}
$$

When $H$ tends towards $0, i^{2 H}$ tends towards 1 . Then, for all lag $k$,

$$
\begin{aligned}
\rho_{\mathrm{dfBm}}(k) & \longrightarrow 1-\frac{n(n-1)}{2 \sum_{k=1}^{n-1}(n-k)} \\
& \longrightarrow 1-\frac{n(n-1)}{2[n(n-1) / 2]} \longrightarrow 0,
\end{aligned}
$$

suggesting that $B_{n}^{H}$ tends towards white noise as $H$ tends towards 0. Accordingly, if one determines, on the basis of (5), the limit values of the autocorrelation function of dfGn when $H$ tends toward 0 [17, page 281], one obtains $\rho_{\mathrm{dfGn}}(0)=1$, $\rho_{\mathrm{dfGn}}(1)=2^{-1}-1=-1 / 2$, and, for $k>1, \rho_{\mathrm{dfGn}}(k)=0$. This autocorrelation function corresponds to that of a differenced white noise [21].

We present in Figures 1(a) and 1(b) the theoretical values of $\rho_{\mathrm{dfGn}}(1)$ and $\rho_{\mathrm{dfBm}}(1)$, obtained from (5) and (20), respectively, for $H$ values ranging from 0 to 1 in both families. This figure shows a clear breakdown in the correlation structures from dfGn to dfBm. As expected, $\rho_{\mathrm{dfGn}}(1)$ starts at -0.5 for $H=0$, and it reaches 1.0 for $H=1 . \rho_{\mathrm{dfBm}}(1)$ is 0 for $H=0$ and grows asymptotically towards 1.0 as $H$ increases.

\section{Discussion}

dfGn and dfBm were originally defined as two distinct families, which can be considered superimposed, with their relationships of summing/differencing. A number of authors, however, have proposed to consider these two families as a continuum, surrounding the mythical border of "ideal" $1 / f$ noise [22-25].

This conception has been favored by the existence of analysis methods which can be applied indifferently on both families and provide continuous metrics for characterizing the series. One of these methods is the Detrended Fluctuation
Analysis, introduced by Peng et al. [26]. In this method, the analyzed series is first summed, and this summed series is divided into nonoverlapping intervals of length $n$. Within each interval, the data are linearly detrended, and the average root mean square $(F(n))$ of this summed and detrended series is computed. Typically, $F(n)$ increases with interval length $n$. A power law is expected, as

$$
F(n) \propto n^{\alpha}
$$

dfGn series are characterized by $\alpha$ exponents ranging from 0 to 1 and $\mathrm{dfBm}$ by exponents ranging from 1 to 2 . Note that the scaling law expressed in (23) just derives from the original definition of $\mathrm{fBm}(1)$. If the series $x_{i}$ is a dfGn, $X_{i}$ is the corresponding $\mathrm{dfBm}$ and $\alpha$ is the Hurst exponent. If $x_{i}$ is a $\mathrm{dfBm}, X_{i}$ belongs to another family of overdiffusive processes, characterized by $\alpha$ exponents ranging from 1 to 2 , and in that case $\alpha=H+1[26]$.

In this methodological framework, $G_{i}^{1}$ and $B_{i}^{0}$ are supposed to be equivalent, both characterized by $\alpha=1$, representing "ideal" $1 / f$ noise, or "pink" noise. From this point of view, dfGn and dfBm are clearly considered linked by a smooth transition, and the $\mathrm{dfGn} / \mathrm{dfBm}$ model is considered as a continuum from roughness to smoothness [22-25, 27]. Das [28] argued that "pink noise is important because this is a kind of threshold between the persistent stable noise $(0.5<H<1,0<\beta<1)$ and the nonstationary noise $\beta>1$ " (page 246). Eke et al. [23] considered $1 / f$ noise as the boundary between $\mathrm{fGn}$ and $\mathrm{fBm}$ families.

In contrast, our present results suggest a clear discontinuity between $\mathrm{dfGn}$ and $\mathrm{dfBm}$, around this supposed $1 / f$ boundary. Note that this breakdown was mainly apparent because we explored correlations up to the limits of the theoretical interval over which $H$ is defined. Most studies that attempted to assess fractal analysis methods on exact signals worked on $\mathrm{dfGn}$ and/or dfBm signals with $H$ exponents ranging from 0.1 to 0.9 , by steps of 0.1 , then excluding the intervals ]0.9, 1[ for dfGn and ]0,0.1[ for dfBm [23, 29]. Figure 1 shows that the breakdown in correlation structure mainly occurs within these intervals, indicated by the vertical dashed lines in both panels, and this could explain why this phenomenon was never reported in the literature. We present in Figure 2 the evolution of the autocorrelation function (up to lag 30), within these two intervals. This figure illustrates the strong divergence of $\mathrm{dfGn}$ and $\mathrm{dfBm}$, when approaching the supposed $1 / f$ boundary.

Stochastic fractal processes can also be defined in the frequency domain, on the basis of a scaling law that relates power (i.e., squared amplitude) to frequency according to an inverse power function, with an exponent $\beta[23,29]$ :

$$
S(f) \propto \frac{1}{f^{\beta}} .
$$

This scaling law defines a family of processes called $1 / f^{\beta}$ processes, which is generally considered equivalent to $\mathrm{dfGn} / \mathrm{dfBm}$ processes. dfGn series correspond to $1 / f^{\beta}$ series with $\beta$ exponents ranging from -1 to 1 and $\mathrm{dfBm}$ to $1 / f^{\beta}$ series with $\beta$ exponents ranging from 1 to 3 , and $\beta$ is linearly 


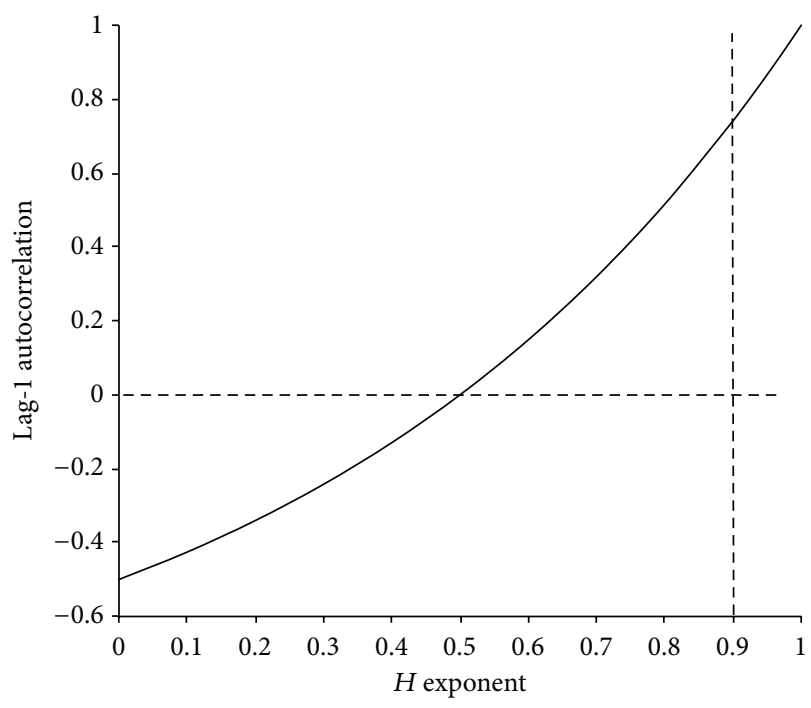

(a)

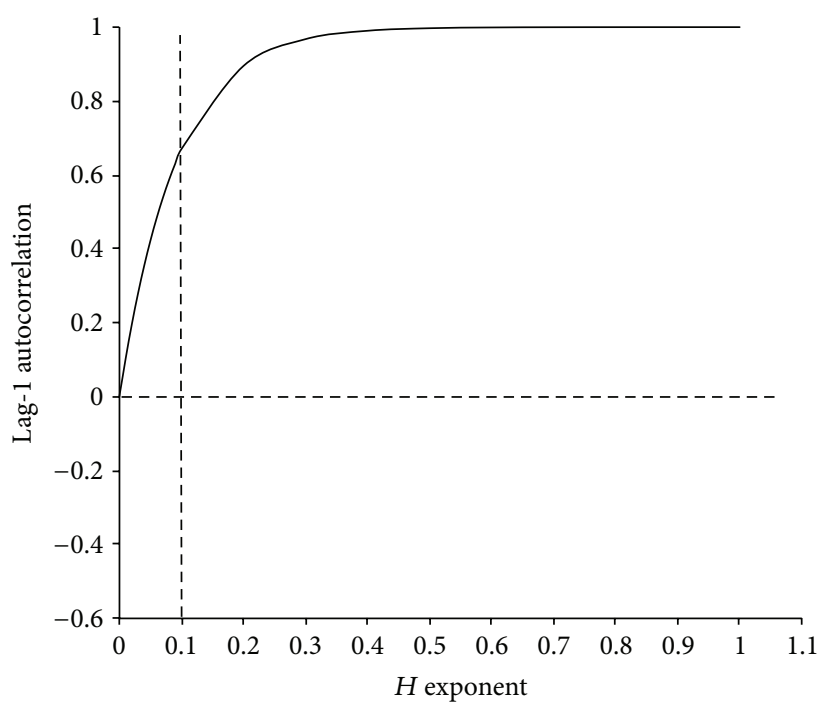

(b)

Figure 1: Theoretical lag-one autocorrelation for $\mathrm{dfGn}(\mathrm{a})$, based on (5), and for $\mathrm{dfBm}$ (b), based on (20), for $H$ values ranging from 0 to 1.

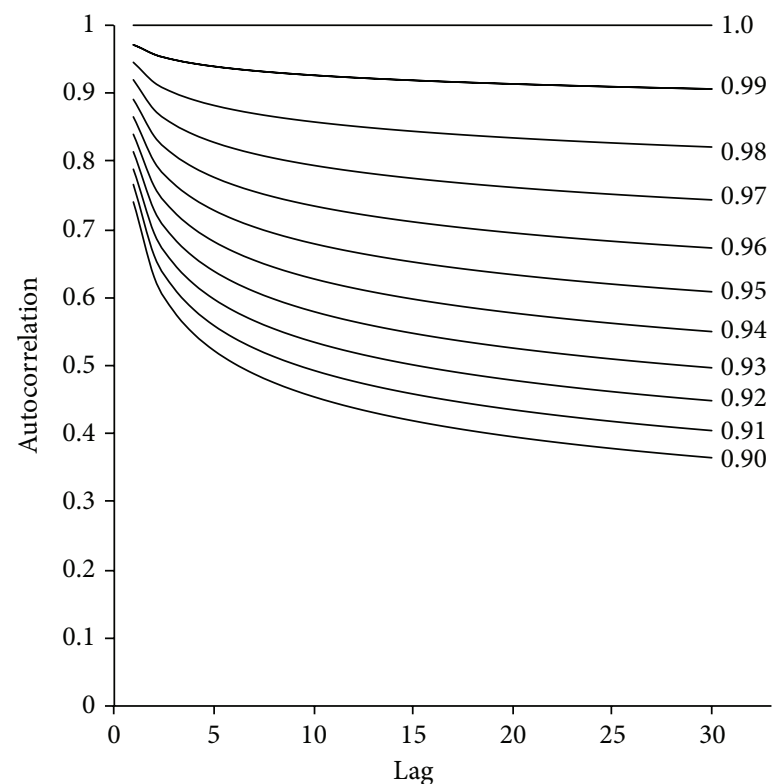

(a)

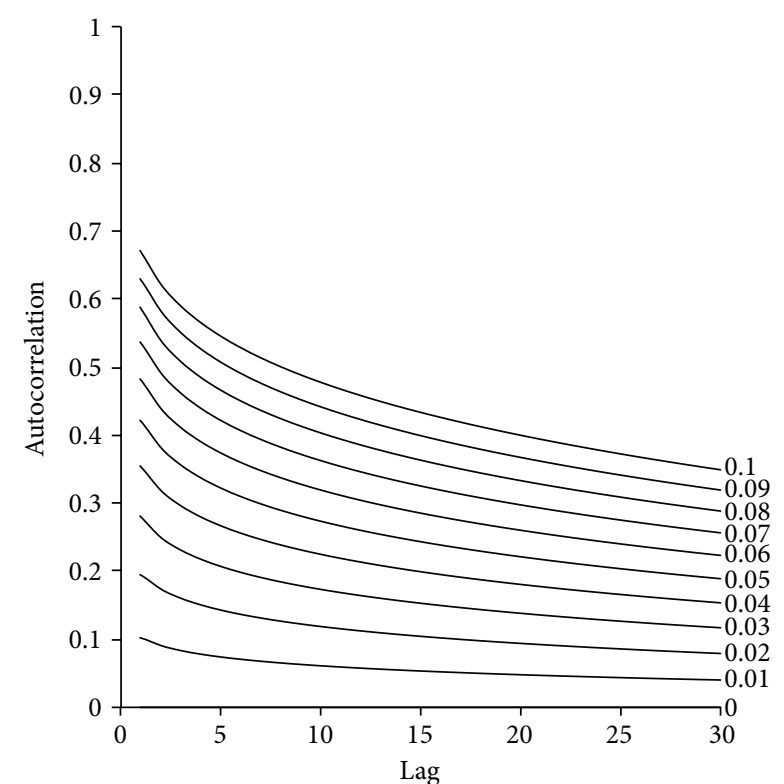

(b)

Figure 2: Theoretical autocorrelation functions, up to lag 30, for $\mathrm{dfGn}$ for $H$ values ranging from 0.9 to 1 (a) and for $\mathrm{dfBm}$ for $H$ values ranging from 0 to $0.1(\mathrm{~b})$.

related to the $\alpha$ exponent: $\beta=2 \alpha-1$ [22]. This linear relationship is generally considered as a logical consequence of the Wiener-Khinchin theorem, which states that the power spectrum of a stationary stochastic process is analogous to the Fourier transform of the corresponding autocorrelation function [30].

For $\beta=1$, power appears proportional to period, a property which was at the origin of the name " $1 / f$ noise." The formal definition of $1 / f^{\beta}$ processes suggests a clear continuity of the correlation properties over the whole range of $\beta$ exponents, and especially around the $1 / f$ boundary. This continuity contrasts with the discontinuity we evidenced in the $\mathrm{dfGn} / \mathrm{dfBm}$ model.

The present paper essentially questions the supposed equivalence between the $\mathrm{dfGn} / \mathrm{dfBm}$ and the $1 / f^{\beta}$ models. It is interesting to note that, within each framework, specific methods have been proposed for generating series with known exponents. Davies and Harte [31] proposed a method for generating dfGn signals with autocorrelation function corresponding to (5), and the spectral synthesis method 
produces $1 / f^{\beta}$ signals with the power law behavior of (24) [32]. However, Eke et al. [23] noted that the Davies-Harte method generated series whose power spectra failed to follow the expected power law of (24) at high frequencies, and conversely for series generated by the spectral synthesis method the autocorrelation tended to zero at high frequencies. These results show that series generated from the $\mathrm{fGn} / \mathrm{fBm}$ or the $1 / f^{\beta}$ frameworks possess different properties. Here we evidence a major difference between the two models: the $1 / f^{\beta}$ model supports the hypothesis of a smooth transition between stationary and nonstationary processes through the $1 / f$ boundary, while the $\mathrm{dfGn} / \mathrm{dfBm}$ model does not.

Beyond its theoretical interest, this result has interesting implications in more applied perspectives. Fractal models currently find useful applications in biomedical engineering, especially for conceiving real-time monitoring devices allowing analyzing physiological fluctuations, for example, in brain activity, heart rate, or gait [33-36]. A deeper understanding of the underlying models is necessary for selecting relevant algorithms of analysis and improving their efficiency. Another important domain is the conception of virtual environments, especially for gait rehabilitation. A number of recent works showed that instructing a patient to synchronize to a fractal environment might induce an enhancement of gait dynamics [37-39]. For that purpose, the generation of appropriate signals, close to $1 / f$ noise, represents an essential step. Considering the behavior of the $\mathrm{dfGn} / \mathrm{dfBm}$ model around the $1 / f$ boundary, one could suggest to favor $1 / f^{\beta}$ inspired methods in such applications.

\section{Conclusion}

This paper highlighted some formal aspects of discrete-time fractional processes, which were rarely considered in the literature, which generally focuses on continuous processes. The derivation of expressions for $\mathrm{dfBm}$ variance (see (18)), scaling coefficient (see (19)), and dfBm autocorrelation function (see (20)) represent at this level useful results.

Our main claim is that the $\mathrm{dfGn} / \mathrm{dfBm}$ model cannot be considered a continuum. This point is clearly evidenced by the formal analysis of the correlation properties of the two classes of processes. dfGn converges towards a straight line process as $H$ reaches its upper limit, $\mathrm{dfBm}$ tends towards white noise as $H$ approaches its lower bound, and neither can be considered as what is commonly referred to as $1 / f$ noise. The supposed continuity between these two classes appears to be just an illusion induced by the methods of analysis that provided continuous metrics, and the concept of "ideal" $1 / f$ noise seems hardly sustainable within this model.

\section{Conflict of Interests}

The author declares that there is no conflict of interests regarding the publication of this paper.

\section{References}

[1] A. L. Goldberger, L. A. N. Amaral, J. M. Hausdorff, P. C. Ivanov, C.-K. Peng, and H. E. Stanley, "Fractal dynamics in physiology: alterations with disease and aging," Proceedings of the National Academy of Sciences of the United States of America, vol. 99, no. 1, pp. 2466-2472, 2002.

[2] S. Wallot, R. Fusaroli, K. Tylén, and E.-M. Jegindø, "Using complexity metrics with R-R intervals and BPM heart rate measures," Frontiers in Physiology, vol. 4, article 211, 2013.

[3] P. Herman, B. G. Sanganahalli, F. Hyder, and A. Eke, "Fractal analysis of spontaneous fluctuations of the BOLD signal in rat brain," NeuroImage, vol. 58, no. 4, pp. 1060-1069, 2011.

[4] T. Montez, S.-S. Poil, B. F. Jones et al., "Altered temporal correlations in parietal alpha and prefrontal theta oscillations in early-stage Alzheimer disease," Proceedings of the National Academy of Sciences of the United States of America, vol. 106, no. 5, pp. 1614-1619, 2009.

[5] P. J. Fadel, S. M. Barman, S. W. Phillips, and G. L. Gebber, "Fractal fluctuations in human respiration," Journal of Applied Physiology, vol. 97, no. 6, pp. 2056-2064, 12004.

[6] M. Latka, M. Glaubic-Latka, D. Latka, and B. J. West, "Fractal rigidity in migraine," Chaos, Solitons and Fractals, vol. 20, no. 1, pp. 165-170, 2004.

[7] J. J. Sosnoff, A. D. Valantine, and K. M. Newell, "The adaptive range of $1 /$ f isometric force production," Journal of Experimental Psychology: Human Perception and Performance, vol. 35, no. 2, pp. 439-446, 2009.

[8] D. G. Stephen and J. Anastas, "Fractal fluctuations in gaze speed visual search," Attention, Perception, and Psychophysics, vol. 73, no. 3, pp. 666-677, 2011.

[9] D. L. Gilden, T. Thornton, and M. W. Mallon, "1/F Noise in human cognition," Science, vol. 267, no. 5205, pp. 1837-1839, 1995.

[10] L. Lemoine, K. Torre, and D. Delignières, “Testing for the presence of $1 / \mathrm{f}$ noise in continuation tapping data," Canadian Journal of Experimental Psychology, vol. 60, no. 4, pp. 247-257, 2006.

[11] K. Torre, D. Delignières, and L. Lemoine, " $1 / \mathrm{f} \beta$ fluctuations in bimanual coordination: an additional challenge for modeling," Experimental Brain Research, vol. 183, no. 2, pp. 225-234, 2007.

[12] J. U. Mafahim, D. Lambert, M. Zare, and P. Grigolini, "Complexity matching in neural networks," New Journal of Physics, vol. 17, Article ID 015003, 2015.

[13] B. B. Mandelbrot and J. W. Van Ness, "Fractional Brownian motions, fractional noises and applications," SIAM Review, vol. 10, no. 4, pp. 422-437, 1968.

[14] B. E. Hurst, "Long-term storage capacity of reservoirs," Transactions of the American Society of Civil Engineers, vol. 116, pp. 770-799, 1951.

[15] M. Matsuzaki, "Fractals in earthquakes," Philosophical Transactions. Series A, Mathematical, Physical, and Engineering Sciences, vol. 348, pp. 449-457, 1994.

[16] W. E. Leland, M. S. Taqqu, W. Willinger, and D. V. Wilson, "On the self-similar nature of Ethernet traffic (extended version)," IEEE/ACM Transactions on Networking, vol. 2, no. 1, pp. 1-15, 1994.

[17] L. A. Lipsitz and A. L. Goldberger, "Loss of 'complexity' and aging: potential applications of fractals and chaos theory to senescence," The Journal of the American Medical Association, vol. 267, no. 13, pp. 1806-1809, 1992.

[18] J. Beran, Statistics for Long-Memory Processes, CRC Press, 1994.

[19] H. Qian, "Fractional Brownian motion and fractional Gaussian noise," in Processes with Long-Range Correlations: Theory and Applications, G. Rangarajan and M. Ding, Eds., vol. 621 of 
Lecture Notes in Physics, pp. 22-33, Springer, Berlin, Germany, 2003.

[20] O. Magre and M. Guglielmi, "Approximation de l'utocorrélation des incréments du (fbm) de Mandelbrot par modélisation de Barnes et Allan," Colloques sur le Traitement du Signal et des Images, vol. 15, pp. 5-8, 1995.

[21] A. M. Wing and A. B. Kristofferson, "Response delays and the timing of discrete motor responses," Perception \& Psychophysics, vol. 14, no. 1, pp. 5-12, 1973.

[22] D. Delignières and V. Marmelat, "Theoretical and methodological issues in serial correlation analysis," in Progress in Motor Control: Neural, Computational and Dynamic Approaches, vol. 782 of Advances in Experimental Medicine and Biology, pp. 127148, Springer, Berlin, Germany, 2013.

[23] A. Eke, P. Hermán, J. B. Bassingthwaighte et al., "Physiological time series: distinguishing fractal noises from motions," Pflügers Archiv-European Journal of Physiology, vol. 439, no. 4, pp. 403415,2000

[24] E. J. Wagenmakers, S. Farrell, and R. Ratcliff, "Estimation and interpretation of $1 / \mathrm{f}($ alpha) noise in human cognition," Psychonomic Bulletin \& Review, vol. 11, pp. 579-615, 2004.

[25] B. J. West, E. L. Geneston, and P. Grigolini, "Maximizing information exchange between complex networks," Physics Reports, vol. 468, no. 1-3, pp. 1-99, 2008.

[26] C.-K. Peng, J. Mietus, J. M. Hausdorff, S. Havlin, H. E. Stanley, and A. L. Goldberger, "Long-range anticorrelations and nonGaussian behavior of the heartbeat," Physical Review Letters, vol. 70, no. 9, pp. 1343-1346, 1993.

[27] V. Marmelat, K. Torre, and D. Delignières, "Relative roughness: an index for testing the suitability of the monofractal model," Frontiers in Physiology, vol. 3, article 208, 2012.

[28] S. Das, Functional Fractional Calculus, Springer, Berlin, Germany, 2011.

[29] D. Delignieres, S. Ramdani, L. Lemoine, K. Torre, M. Fortes, and G. Ninot, "Fractal analyses for 'short' time series: a reassessment of classical methods," Journal of Mathematical Psychology, vol. 50, no. 6, pp. 525-544, 2006.

[30] J. W. Kantelhardt, "Fractal and multifractal time series," in Mathematics of Complexity and Dynamical Systems, R. A. Meyers, Ed., pp. 463-487, Springer, Berlin, Germany, 2011.

[31] R. B. Davies and D. S. Harte, "Tests for hurst effect," Biometrika, vol. 74, no. 1, pp. 95-101, 1987.

[32] D. Saupe, "Algorithms for random fractals," in The Science of Fractal Images, H. O. Peitgen and D. Saupe, Eds., pp. 71-136, Springer, Berlin, Germany, 1988.

[33] A. F. Farag, S. M. El-Metwally, and A. A. A. Morsy, "Automated sleep staging using detrended fluctuation analysis of sleep EEG," in Soft Computing Applications, V. E. Balas, J. Fodor, A. R. Várkonyi-Kóczy, J. Dombi, and L. C. Jain, Eds., Advances in Intelligent Systems and Computing, pp. 501-510, Springer, Berlin, Germany, 2013.

[34] R.-G. Yeh, J.-S. Shieh, Y.-Y. Han, Y.-J. Wang, and S.-C. Tseng, "Detrended fluctuation analyses of short-term heart rate variability in surgical intensive care units," Biomedical Engineering-Applications, Basis and Communications, vol. 18, no. 2, pp. 67-72, 2006.

[35] A. Hartmann, P. Mukli, Z. Nagy, L. Kocsis, P. Hermán, and A. Eke, "Real-time fractal signal processing in the time domain," Physica A: Statistical Mechanics and its Applications, vol. 392, no. 1, pp. 89-102, 2013.
[36] C. Kamath, "A new approach to detect congestive heart failure using sequential spectrum of electrocardiogram signals," Medical Engineering and Physics, vol. 34, no. 10, pp. 1503-1509, 2012.

[37] A. K. Kiefer, C. K. Rhéa, and W. H. Warren, "VR-based assessment and rehabilitation of functional mobility," in Human Walking in Virtual Environments, F. Steinicke, Y. Visell, J. Campos, and A. Lecuyer, Eds., pp. 333-350, Springer, Berlin, Germany, 2013.

[38] D. Katsavelis, M. Mukherjee, L. Decker, and N. Stergiou, "The effect of virtual reality on gait variability", Nonlinear Dynamics, Psychology, and Life Sciences, vol. 14, no. 3, pp. 239-256, 2010.

[39] C. K. Rhea, A. W. Kiefer, M. W. Wittstein et al., "Fractal gait patterns are retained after entrainment to a fractal stimulus," PLoS ONE, vol. 9, no. 9, Article ID e106755, 2014. 


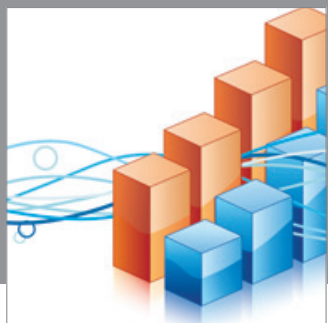

Advances in

Operations Research

mansans

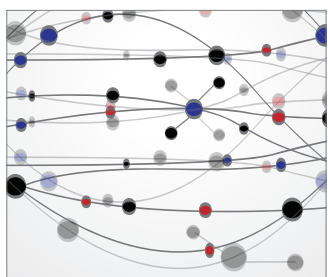

The Scientific World Journal
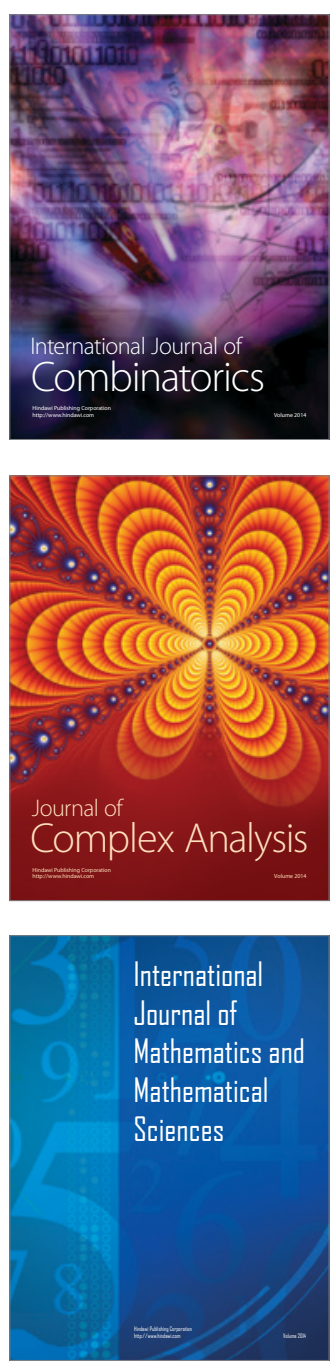
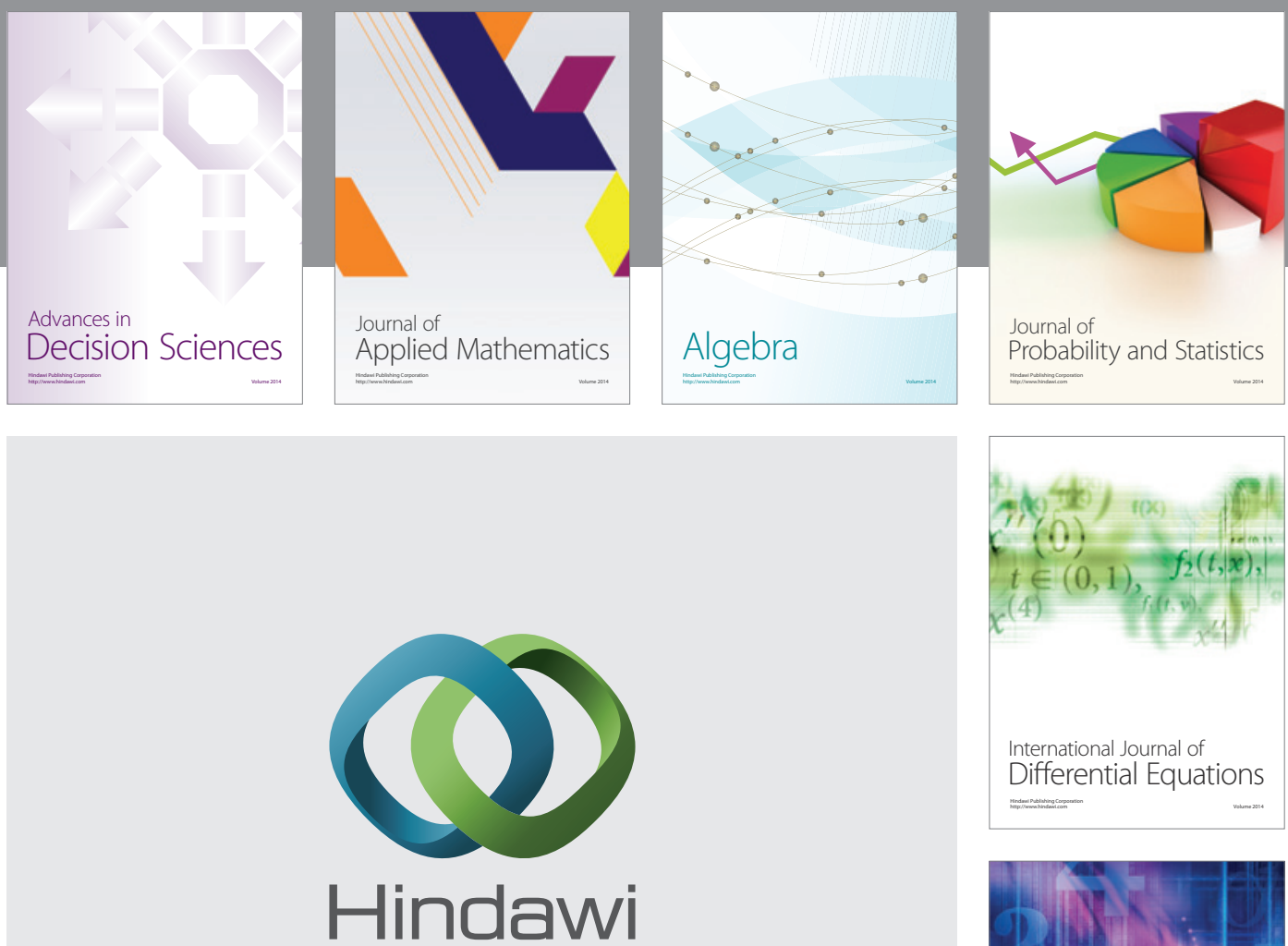

Submit your manuscripts at http://www.hindawi.com
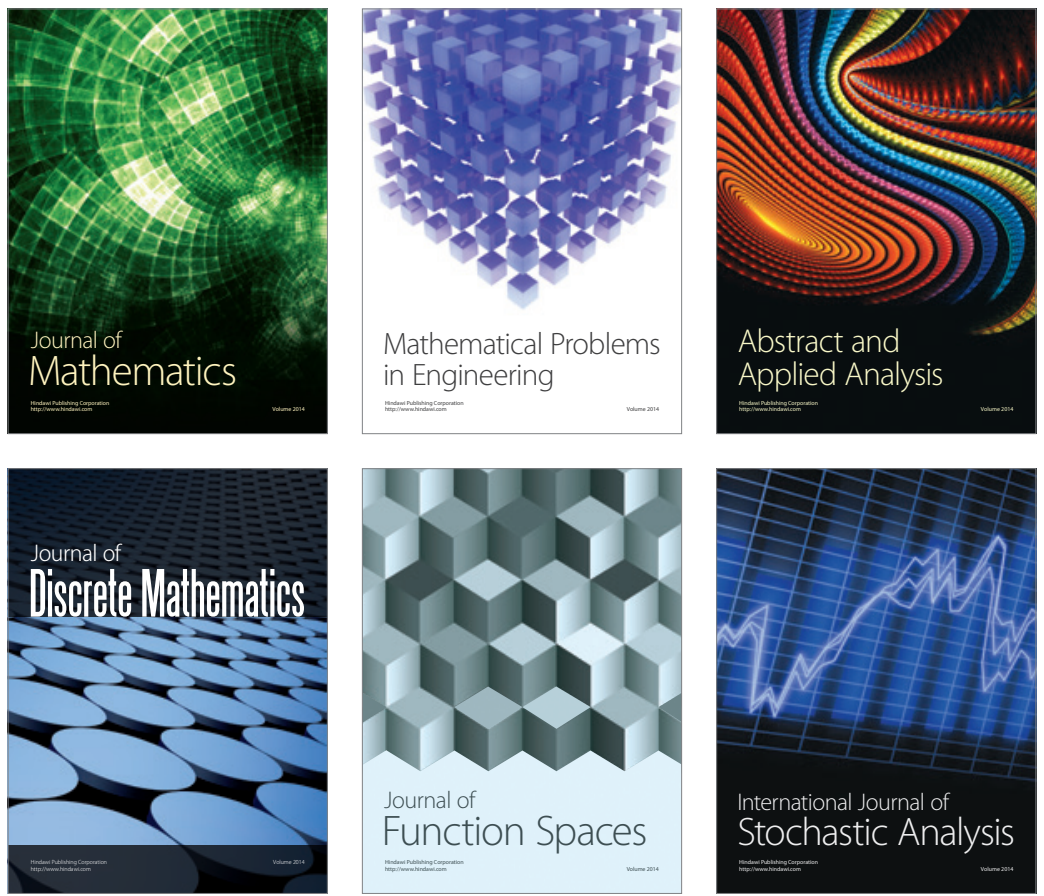

Journal of

Function Spaces

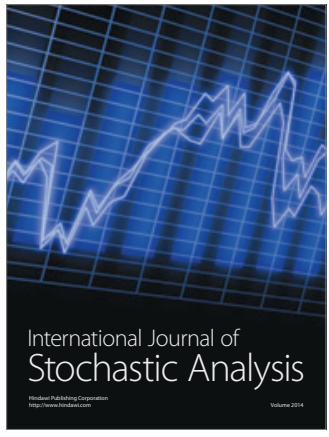

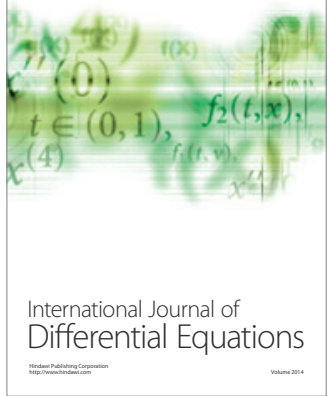
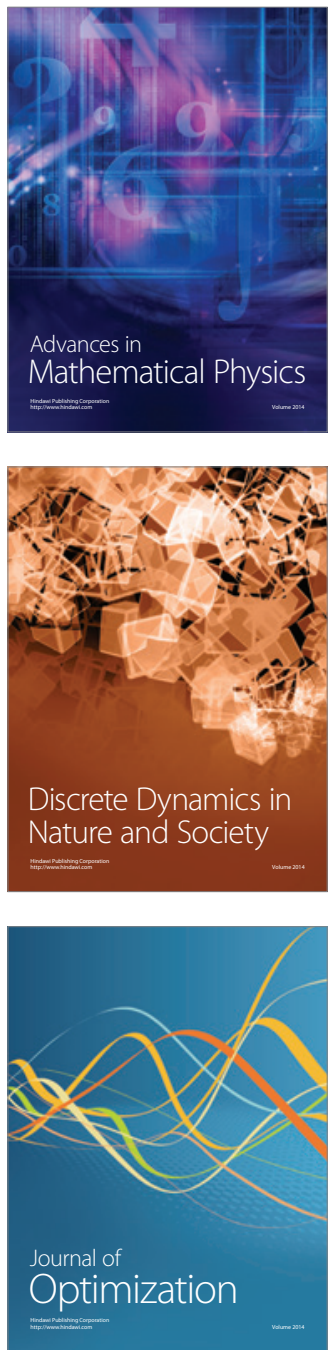